\title{
Größenprogredientes Ulkus infraaurikulär links
}

Broglie, M A ; Tinguely, M ; Stenner, F ; Stöckli, S J

DOI: https://doi.org/10.1007/s00106-008-1744-8

Other titles: A progressive ulcerative neck mass on the left infraauricular area

Posted at the Zurich Open Repository and Archive, University of Zurich ZORA URL: https://doi.org/10.5167/uzh-10907

Journal Article

Published Version

Originally published at:

Broglie, M A; Tinguely, M; Stenner, F; Stöckli, S J (2008). Größenprogredientes Ulkus infraaurikulär links. HNO, 56(10):1054-1056.

DOI: https://doi.org/10.1007/s00106-008-1744-8 
HNO 2008 · 56:1054-1056

DOI 10.1007/s00106-008-1744-8

Online publiziert: 11. April 2008

(c) Springer Medizin Verlag 2008

M.A. Broglie ${ }^{1} \cdot$ M. Tinguely $^{2} \cdot$ F. Stenner ${ }^{3} \cdot$ S.J. Stöckli ${ }^{1}$

${ }^{1}$ Klinik für Ohren-, Nasen-, Hals- und Gesichtschirurgie, Universitätsspital Zürich

${ }^{2}$ Institut für klinische Pathologie, Universitätsspital Zürich

${ }^{3}$ Klinik für Medizinische Onkologie, Universitätsspital Zürich

\section{Größenprogredientes Ulkus infraaurikulär links}

\section{Anamnese}

Eine 83-jährige Patientin wurde uns von einem niedergelassenen HNO-Arzt aufgrund eines seit 3 Monaten bestehenden, an Größe zunehmenden Ulkus infraaurikulär links zugewiesen. Bei Erkrankungsbeginn fand sich eine Rötung und Schwellung mit fötider Sekretion zervikal links, welche anfänglich gut auf eine Antibiotikatherapie ansprach. Im weiteren Verlauf entwickelte sich dann aber eine progrediente Exulzeration der Haut. Aufgrund von ausgeprägten Komorbiditäten und dem Wunsch der Patientin, auf Interventionen und Therapien möglichst zu verzichten, wurden eingangs keine weiteren Abklärungen durchgeführt. Die Zuweisung erfolgte schlussendlich aufgrund starker Schmerzen und einer aus pflegerischen Gründen unhaltbaren Situation.

Anamnestisch bekannt war ein 1999 diagnostiziertes Hodgkin-Lymphom Stadium III B mit Zustand nach Chemotherapie (2 Zyklen MVPP 04/oo, 3. Zyklus o6/oo) und klinischer Remission seitdem.

\section{Klinischer Befund}

Infraaurikulär links zeigte sich ein etwa $4 \times 2 \mathrm{~cm}$ großes Ulkus mit direkter Sicht auf den freiliegenden M. sternocleidomastoideus sowie den Unterpol der Gl. parotis. Die Wundhöhle ließ sich bis zur Gl. submandibularis verfolgen. Sie war teilweise angefüllt mit einem trüben, zähflüssigen Sekret. Ein Fistelgang war nicht darstellbar. Der Hautrand war etwas aufgeworfen. Die Haut zeigte bis auf eine aktinische Keratose an der Stirn keine pathologischen Läsionen. Der restliche HNO-Status war unauffällig (• Abb. 1).

\section{Diagnostik}

Im Wundabstrich zeigte sich eine Kontamination mit Staphylococcus aureus ohne Nachweis von Pilzen oder Mykobakterien.

Das Ergebnis der Feinnadelpunktion mit Biopsien und deren Histologie ist - Abb. 2 und 3 zu entnehmen.
Liebe Kolleginnen und Kollegen, hier kann auch Ihr Fall

dargestellt werden!

Haben Sie Anregungen oder eine interessante Falldarstellung? Reichen Sie diese bitte über das Online-System „Editorial Manager" ein. Informationen dazu auf der Zeitschriftenhomepage www.HNO.springer.de unter dem Navigationspunkt "Für Autoren".

Rubrikherausgeber:

Prof. Dr. F. Bootz

Universitäts-HNO-Klinik Bonn

friedrich.bootz@ukb.uni-bonn.de 
Abb. 1 - Ausgedehntes Ulkus infraaurikulär links mit Blick auf den Unterpol der Gl. parotis sowie den freiliegenden M. sternocleidomastoideus. In der Bildmitte ist ein Lymphknoten ersichtlich. Der kaudale Anteil der Ohrmuschel ist angeschnitten

Abb. 2 - Große Lymphoblasten mit oval bis rund geformten, vesikulären Nuklei, die feines Chromatin und 1 bis 3 Nukleoli aufweisen. Es sind verstreut reaktive Lymphozyten sichtbar (Vergr. 400:1, HE, Inset: Giemsa-Färbung)

Abb. 3 Immunhistochemisch gefärbte Zellen exprimieren $\mathrm{CD} 20$ (CD20-IHC-Färbung, Vergr. 400:1)
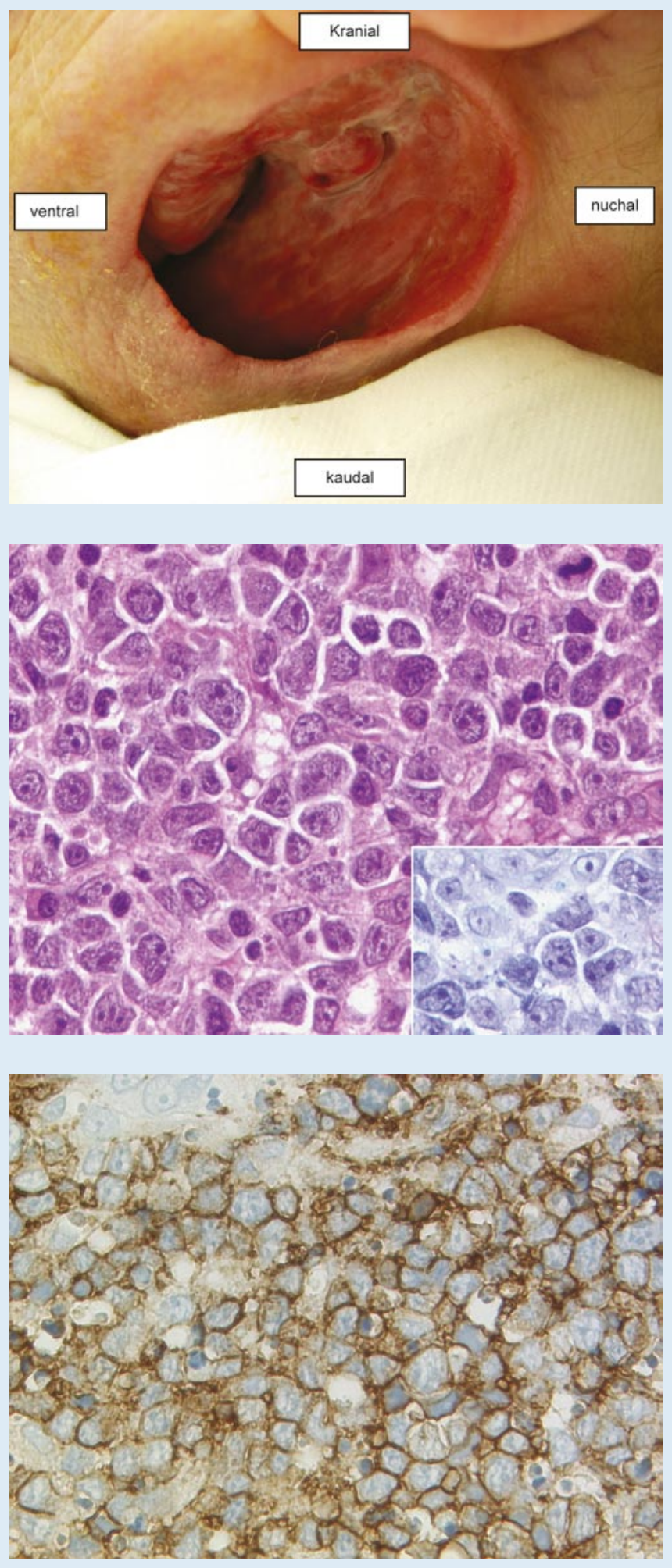


\section{\ Diagnose: Diffuses großzelliges B-Zell-Lymphom}

Histologisch zeigte das Lymphom ein dichtes, überwiegend aus Zentroblasten und wenigen Immunoblasten bestehendes Infiltrat (• Abb. 2). Immunhistochemisch exprimierten die Tumorzellen stark den B-Zell-Marker CD2o (• Abb. 3). Der gleichzeitige Nachweis von CD10 und Bcl-6 wies auf einen Keimzentrumsursprung des Lymphoms hin. Bcl-2 war partiell exprimiert. Die Proliferationsrate war stark erhöht und lag bei nahezu $100 \%$ (Mib1), was den aggressiven Charakter dieser Läsion unterstrich.

Unter Zuhilfenahme molekularer Untersuchungen mittels Interphasen-Fluoreszenz-in-situ-Hybridisierung (FISH) konnte keine Translokation $\mathrm{t}(14 ; 18), \mathrm{t}(8 ; 14)$ oder $\mathrm{t}(3 ; 14)$ nachgewiesen werden.

Weder die Morphologie noch die $\mathrm{Zu}-$ satzuntersuchungen erlaubten es, dieses Lymphom einem primär kutanen Lymphom zuzuordnen. Da auf weitergehende Untersuchungen verzichtet wurde (z. B. Knochenmarkuntersuchung), konnte aus hämatopathologischer Sicht keine Aussage über die Ausdehnung oder den definitiven Ursprung gemacht werden.

\section{Definition}

Die diffus großzelligen B-Zell-Lymphome (DLBCL) sind die häufigsten Non-Hodgkin-Lymphome (>30\% aller NHL). Pathognomonisch zeigen sich proliferierende große, neoplastische B-Zellen, deren Kern mehr als doppelt so groß ist wie der eines normalen kleinen Lymphozyten. Morphologisch lassen sich verschiedene Formen von DLBCL unterscheiden. Immunphänomenologisch exprimieren diese Lymphome im Gewebe die B-Zell-Marker $\mathrm{CD}_{2} \mathrm{O}$ und $\mathrm{CD} 79$ a sowie variabel weitere Proteine, welche die histogenetische Zuordnung erleichtern. Typischerweise findet sich ein hoher Proliferationsindex. Anders als bei den TZell-Lymphomen, die häufig mit kutanem Befall assoziiert sind, ist bei den diffus großzelligen B-Zell-Lymphomen ein primärer Hautbefall eher selten. Typischerweise treten kutane B-Zell-Lymphome bei älteren Patienten (Durchschnittsalter 72 Jahre) in Gestalt rötlich-livider Knoten hauptsächlich an der linken unteren Extremität auf [1].

\section{Verlauf}

Diffus großzellige B-Zell-Lymphome haben unbehandelt eine schlechte Prognose. Bei adäquater Immun- und Chemotherapie ist die Prognose dieser Lymphome durch einen sog. internationalen Prognoseindex (IPI) einzuschätzen. Diesem Score liegen klinisch einfach zu bestimmende Parameter wie Alter, Allgemeinzustand, Ausbreitungsgrad des Lymphoms, Zahl der Extranodalbefälle sowie Standardlaborparameter, z. B. die Höhe der Laktatdehydrogenase (LDH) zugrunde [2]. Basierend auf dem IPI unterscheidet man 4 prognostische Gruppen: a) niedriges, b) niedrig-intermediäres, c) hoch intermediäres und d) hohes Risiko mit jeweiligen Jahresüberlebensraten von $73 \%, 50 \%, 43 \%$ und $26 \%$ [3]. Der IPI-Score hat in mehreren prospektiven Studien seine klinischprognostische Wertigkeit bewiesen und sollte heute Grundlage der Therapieentscheidung sein.

\section{Therapie}

Bei dem hier vorliegenden Fall eines diffus großzelligen B-Zell-Lymphoms ist eine systemische Immun- und Chemotherapie mit einem Rituximab-CHOP oder vergleichbarem Schema indiziert. Anders als bei primären kutanen B-Zell-Lymphomen ist eine Radiotherapie nicht ausreichend, da es sich um eine systemische Erkrankung handelt. Der Allgemeinzustand der Patientin ließ keine adäquate Therapie $\mathrm{zu}$ und eine palliativ intendierte Therapie mit Steroiden hatte, wie erwartet, neben einer kurzfristigen symptomatischen Besserung keinen Einfluss auf den klinischen Verlauf. Die Patientin starb innerhalb von wenigen Wochen nach Diagnosestellung.

\section{Diskussion}

Zervikale Hautabszesse sind in der HNOärztlichen Praxis ein häufiges Bild. Beim Versagen einer antibiotischen Therapie sollte eine genauere klinische und laborchemische Untersuchung erfolgen. Immunkompromittierende Faktoren wie Diabetes mellitus müssen ebenso ausgeschlos- sen werden wie Antibiotikaresistenzen, atypische Erreger (Pilzinfekt, Mykobakterien) oder Neoplasien. Dafür sind mehrere tiefe Biopsien nötig. Auffallend an diesem Ulkus war die ausgeprägte Zerstörung der Kutis und Subkutis unter Respektierung von Gewebegrenzen wie der Muskulatur und des Drüsengewebes der Gl. parotis sowie die nur geringen soliden Anteile.

Dies sprach eher gegen das Vorliegen einer Lymphknotenmetastase eines Plattenepithelkarzinoms. In • Abb. 1 sieht man in der Tiefe Strukturen, die makroskopisch und histologisch Lymphknoten entsprechen. Sieben Jahre nach einem Hodgkin-Lymphom dachten wir an ein Spätrezidiv mit ungewöhnlicher Präsentation. Das schließlich bioptisch diagnostizierte diffuse großzellige B-Zell-Lymphom stand aber mit diesem in keinem Zusammenhang.

In dem vorliegenden Fall kann man davon ausgehen, dass es nicht primär, sondern eher sekundär im Sinne von trophischen Störungen durch die Beeinträchtigung der kutanen Vaskularisation zu einer Affektion der Haut durch das Lymphom gekommen war.

\section{Korrespondenzadresse \\ Dr. M.A. Broglie}

Klinik für Ohren-, Nasen-, Hals- und Gesichtschirurgie, Universitätsspital Zürich, Frauenklinikstrasse 24, 8091 Zürich, Schweiz martina.broglie@usz.ch

Interessenkonflikt. Der korrespondierende Autor gibt an, dass kein Interessenkonflikt besteht.

\section{Literatur}

1. Beyeler M, Burg G, Dummer R (2004) Kutane Lymphome. Hautarzt 55: 1005-1017

2. Shipp MA (1997) Can we improve upon the international Index? Ann Oncol (Suppl 1) 8: 43-47

3. Shipp MA, Harrington DP, Anderson JR (1993) A prognostic model for aggressive Non-Hodgkin's Lymphoma. N Engl J Med 329: 987-994

4. Zeltser R, Kalra A, Stadecker MJ, Krejici-Papa N (2006) Unusual presentation of B-cell-Lymphoma as a cutaneous abscess. J Am Acad Dermatol (Suppl 2) 55: S24-S27

5. Kim BK, Surti U, Pandya AG, Swerdlow SH (2003) Primary and secondary cutaneous diffuse large BCell-Lymphomas. Am J Surg Pathol 27: 356-364

6. Cogliatti SB, Novak U, Henz S et al. (2006) Diagnosis of Burkitt Lymphoma in due time: a practical approach. Br J Haematol 134: 294-301 